\title{
A Framework for Intelligent Condition-based Maintenance of Rotating Equipment using Mechanical Condition Monitoring
}

\author{
Mohammadreza Tahan B. ${ }^{1, a}$, Masdi Muhammad ${ }^{1}$ and Z. A. Abdul Karim ${ }^{1}$ \\ ${ }^{1}$ Department of Mechanical Engineering, Universiti Teknologi PETRONAS, Bandar Seri Iskandar, \\ 31750 Tronoh, Perak, Malaysia
}

\begin{abstract}
The ideal end result of maintenance strategy is to increase profitability, improve product quality and ensure safety conditions. In condition-based maintenance (CBM), asset health is monitored regularly to maximize reliability and availability by determining necessary maintenance at the right time. Review of recent studies shows most of developed approaches propose a standalone system for each stage of maintenance system. In order to standardize a generic architecture for machinery CBM, this paper attempts to introduce an intelligent framework consisting of several functional modules, starting from data acquisition and ending to advisory generation, with the emphasis on approaches of condition monitoring and maintenance decision-making.
\end{abstract}

\section{Introduction}

Operational safety, asset availability and effective maintenance cost have a tremendous influence on the competitiveness of companies and organizations. Since rotating machinery are the heart of production systems in a wide variety of industrial applications, their minor malfunctioning may cause a massive breakdown of the entire plant. Today's industry uses increasingly complex machines, required to last for longer time and extreme demanding performance criteria. Although recent progress lead to higher efficiency and enhance longevity of rotating machinery, they are still vulnerable to various problems. According to Figure 1 [1], annual cost of seven major rotating equipment are ranked between the ten largest of all industrial equipment. In order to facilitate the implementation of an efficient maintenance system for rotating machinery, the main purpose of current paper is to introduce a generic intelligent maintenance framework by using mechanical condition monitoring.

- 38\%: Boilers and unfired pressure vessels

-36\%: Turbomachinary and reciprocating equipment

- 12\%: Piping

6\%: Non rotating electrical equipment

$\square \%$ : Fired heaters and fired pressure vessels

$3 \%$ : Other production machinery

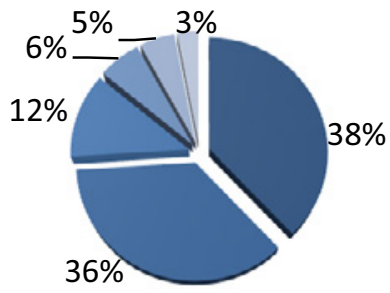

Figure 1. Loss represented by six industrial equipment categories.

\footnotetext{
${ }^{\text {a }}$ Corresponding author : mrtahan@yahoo.com
}

This is an Open Access article distributed under the terms of the Creative Commons Attribution License 2.0, which permits unrestricted use, distribution, and reproduction in any medium, provided the original work is properly cited. 


\section{Advantages of Condition-Based Maintenance}

Maintenance is described as a collection of activities to restitute performance of an equipment to its designated functions. To minimize the operation and maintenance cost of machinery, employment of an efficient maintenance strategy is necessary. One of the effective ways to improve system availability and reduce maintenance cost is to migrate from costive fail and fix reactive-based maintenance into predict and prevent condition-based maintenance. Various aspects of maintenance strategies, emphasizing the benefits of predictive condition-based maintenance is listed in Figure 2.

\begin{tabular}{|c|c|c|}
\hline $\begin{array}{l}\text { Category } 1 \\
\text { Corrective maintenance } \\
\text { - Fail and Fix strategy } \\
\text { - Reactive-based maintenance } \\
\text { - Unscheduled and upon } \\
\text { failure/work stoppage } \\
\text { - No need to data collection } \\
\text { - Capable to restore the functional } \\
\text { abilities of failed components by } \\
\text { either repairing the defect or } \\
\text { replacing the new ones } \\
\text { - Equipment availability and } \\
\text { reliability is unpredicatble }\end{array}$ & $\begin{array}{l}\text { Category } 2 \\
\text { Preventive maintenance } \\
\text { - Prevent strategy } \\
\text { - Time-based maintenance } \\
\text { - Scheduled at regular time } \\
\text { intervals based on failure history } \\
\text { or test data } \\
\text { - Manual data collection } \\
\text { - Prevent the functional failures by } \\
\text { replacing critical components } \\
\text { - Equipment may be overhauled } \\
\text { when they are still in a very good } \\
\text { condition or may fail before a } \\
\text { scheduled overhaul, therefore } \\
\text { availability may drop }\end{array}$ & $\begin{array}{l}\text { Category3 } \\
\text { Predictive maintenance } \\
\text { - Predict \& Prevent strategy } \\
\text { - Condition-based maintenance } \\
\text { " Just-in-time based on mechanical } \\
\text { /performance deterioration analysis } \\
\text { - On-line (real time and continuous) or } \\
\text { off-line (periodic) monitoring and } \\
\text { data collection } \\
\text { - equipment operating conditions are } \\
\text { continuously monitored to identify } \\
\text { the need for maintenance in real time } \\
\text { - Leads to highest equipment's } \\
\text { availability and reliability }\end{array}$ \\
\hline
\end{tabular}

Figure 2. Various aspects of maintenance strategies.

\section{Framework of Intelligent CBM using Mechanical Condition Monitoring}

The principal concept of CBM is to use equipment deterioration information extracted and featured from sensing and data processing to minimize downtime of the system by prognostics and diagnostics [2]. To do so, an intelligent CBM framework for machinery equipment consists of several key steps, mainly including the following, is suggested in Figure 3.

- Data acquisition: Provides access to digitized sensors or transducers to obtain helpful information (data collection), use network to carry information (data communication), and information transfer systems to store the data and provide fundamental for next steps (data storage and handling).

- Data pre-processing: Cleans and filters the measured information from noise and bias before analysis to reach more reliable monitoring results. Noise is the difference in amount between repeated measurements of an identical item and sensor bias is the fixed error that remains steady.

- Feature extraxtion: To generate, represent and select the best sensor features in order to find out the most significant and reliable feature combinations for a specific monitoring purpose.

- Health assesment: Health assessment is the process of determining health status and the equipment deterioration using information provided by condition monitoring. This step provides the unsatisfactory level of operation or the failure and breakdown threshold of asset.

- Prognostic: Prognostics is the ability to provide early finding of the precursor/incipient fault of a component, and to have the means and technology to predict the progress of this defect.

- Diagnostic: Fault detection to indicate something is going wrong, identification to root cause of the fault and isolation to locate the faulty component are three major tasks of diagnostic.

$\mathrm{CM}$ of the industrial equipment can be extinsively classified into mechanical and performance condition monitoring [3]. In performance health monitoring, faults such as a surge, fouling, etc., leads to changes in equipment performance parameters like flow-rate and efficiency. This in turn produce changes in observable parameters such as pressure, temperature, fuel flowrate and rotational speeds. 
The deviated performance reflected from these acquired data can be used to detect and isolate component faults. On the other hand, in mechanical condition monitoring the equipment degrades due to deterioration in mechanical properties that affect the dynamic characteristics of components. There are various techniques to assess this mechanical health deterioration. Figure 4 lists the most effective mechanical monitoring approaches. Recent evidence shows that combining two or more of condition monitoring techniques provides greater and more reliable information.

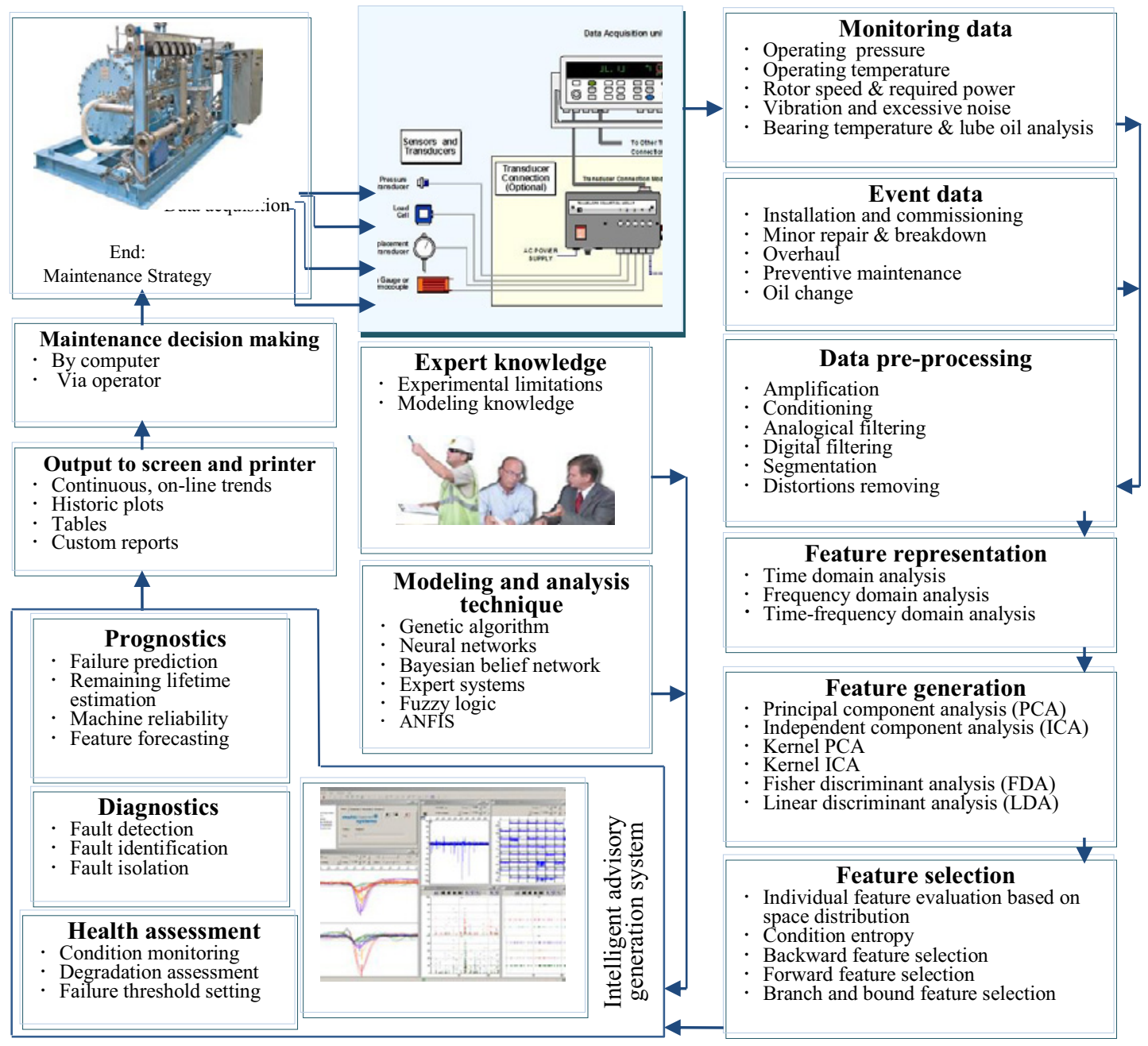

Figure 3. Framework of intelligent CBM using mechanical condition monitoring.

According to [4], for intelligent advisory generation system, most of the investigated prognostics and diagnostic approaches can be categorized into three main types: physics-based, statistical and artificial intelligence. In physics-based models such as Kalman filter, it is necessary to develop models mathematically to combine defect increase formulas, equipment operational knowledge, and condition monitoring data to reach "knowledge-rich" result. For most equipment, this kind of approaches may not be the most applicable method when the considered failure type is frequently unique and is tough to be specified without interrupting operation. Beside physics-based methods, statistical approaches also have been investigated. The principle of these approaches is to compare the signal from faulty equipment with a reference, demonstrating the healthy condition to check whether the value is in safe limits or not. The success of this approach depends crucially to the chosen reference data and reaching to useful information is doubtful. 


\section{Vibration Analysis}

This approach is very popular and well accepted in plants to detect faults at the early stage. As a general rule, machine doesn't break down or fail without some form of warning, which is indicating by increasing vibration level. The frequency range typically from approximately $1 \mathrm{~Hz}$ to near $20 \mathrm{kHz}$.

- Mainly to track: Mass unbalance, Shaft bent, Bow or rub, Misalignment and preloads, Crack, Fluid induced instability, Mechanical looseness and Bearing assembly looseness.

- Merits: Capable to diagnose an extensive range of faults/failure in rotating machinery and Very effective at detecting resonance.

- Limitation: Comparatively expensive and Unable to monitor low speed machinery.

\section{Oil analysis}

Oil and wear debris analysis have proven in many cases a leading indicator of wear mechanisms and wear modes in industrial machinery.

- Mainly to track: Adhesion and abrasion, Corrosion and erosion, Fatigue and Fretting.

- Merits: Provide further insight about the wear rate and Required lower initial investment in comparison to the other methods.

- Limitations: It's diagnostic ability is mostly limited to faults related to wear.

\section{Thermography}

Thermography is the process of using a thermal imager to detect emitted heat of objects. The technology allows operators to validate normal operations and, locate thermal anomalies which indicate possible faults.

- Mainly to track: Any fault that lead to temperature increase in components including: Friction \& cracks, Mass unbalance, Shaft bent or bow, Misalignment and preloads, Bearing assembly looseness, Unsuitable lubrication and Electricals faults.

- Merits: Simple, quick and efficient screening tool since is a non-contact remote sensing.

- Limitations: Late warning of impending failure in comparison to vibration based methods, Extensive working experience about the faulty equipment and sufficient heat transfer knowledge is required.

\section{Ultrasonic inspection}

In this approach, recorded high frequency emissions are electronically translated down and analyzed for enhanced diagnostics. Since ultrasound wave lengths are magnitudes smaller, this approach is much more conducive to locating and isolating the source of problems in loud plant environments. Related frequency ranges from $20 \mathrm{kHz}$ to approximately $80 \mathrm{kHz}$.

- Mainly to track: Leak detection, Bearing condition, Lack of lubrication, Over lubrication, Ionization, Cavitations, Fault analysis of compressor's valve and Testing for arcing and corona in electrical apparatus.

- Merits: Tends to be highly localized, capable for operating in loud and noisy environments, Capable to be used in slow-speed machines, Lower cost in contrast with vibration analysis.

- Limitation: Limited diagnostic ability in comparison with vibration analysis, More reliable result is achievable when used as a complementary approach in an integrated package of conditioning monitoring techniques.

\section{Acoustic emission}

Acoustic emissions are the sound waves or stress waves generated when a piece of material undergoes stress due to external forces. These waves can be measured to detect where the stress has caused wear or degradation, including crushing, cracking or any kind of impacts. Frequency range begins after ultrasonic vibration and continues nearly to $1 \mathrm{MHz}$.

- Mainly to track: Friction and wear, Leakage, Lack of lubrication, Bearing assembly looseness, Cracking, Spalling and Cyclic fatigue.

- Merits: Earlier detection in comparison to vibration analysis, No spectral overlap with mechanical vibration, Not affected by the mechanical noise from adjacent machinery or structural resonances and Only one AE sensor is sufficient.

- Limitations: Signal attenuation may affect the results and Difficult to process, interpret and classify the intelligent information from the acquired $\mathrm{AE}$ data.

Figure 4. A comparison between various types of mechanical condition monitoring techniques. 
On the other hand, artificial intelligence (AI) approaches mostly rely on real-time or collected historical data from the sensors and measurements and do not require a detailed mathematical model of the machinery. As noted by [5], The most well-known artificial intelligence techniques for machinery diagnosis and prognostics are Genetic Algorithms (GA), Artificial Neural Networks (ANN), Expert Systems (ES), Fuzzy Logic System (FLS), and Adaptive neuro-fuzzy inference system (ANFIS). A summary of artificial intelligence methods that have been used and reported in the literature for failure monitoring of rotating machinery is given in Table 1. Finding from literature shows using both learning power of ANN and reasoning of FL in form of ANFIS offers an appealingly powerful platform for data analysis and advisory generation.

Table 1. Comparison of prognostic/diagnostic modelling and analysis approaches

\begin{tabular}{|l|c|c|c|c|c|}
\hline & GA & ANN & ES & FLS & ANFIS \\
\hline Model complexity & Fairly high & Fairly high & High & High & High \\
\hline Computation speed & Low & High & High & Fairly high & Fairly high \\
\hline Coping noise and sensor bias & Yes & Yes & Yes & Yes & Yes \\
\hline Capable of data fusion & No & Yes & Yes & Yes & Yes \\
\hline Real time analysis & No & Yes & No & Yes & Yes \\
\hline Block box deficiency & No & Yes & No & No & No \\
\hline Expert knowledge capability & No & No & Yes & Yes & Yes \\
\hline
\end{tabular}

\section{Concluding Remarks and Outlook on Future Challenge}

In order to easily implement prognostics and diagnostics for rotating equipment, this study developed a framework for intelligent condition-based maintenance system using mechanical health monitoring. This platform includes essential functional layer and modules to facilitate implementation of CBM system for machinery, comprising software and hardware requirments. To suggest this basic framework, three principal steps are discussed: (1) Architecture to develop the CBM system, (2) A comparison of mechanical condition monitoring main categories, and (3) Introduction of most popular modeling and analysis method. It should also be noted that the major concerns facing in implementation of condition-based maintenance for rotating machinery definitely continue to be: a) Selection of effective data acquisition sensors, b) Impressive signal pre-processing and feature extraction methods, c) Utilizing expert knowledge and suitable user-friendliness feature for analysis, d) Design of an effective data fusion model to combine all partial data from various sensors, f) Improve the ability to integrate as many monitoring approaches as possible.

\section{Acknowledgements}

The authors are grateful for the funding and facilities support by Universiti Teknologi PETRONAS.

\section{References}

1. E. E. Clark, 25th Turbomachinery Symposium, Texas A\&M University, College Station, TX, 103122 (1996).

2. J. Lee, J. Ni, D. Djurdjanovic, H. Qiu, and H. Liao, Comput. Ind., 57, 476-489 (2006).

3. S. G. Barad, P. V. Ramaiah, R. K. Giridhar, and G. Krishnaiah, Mech. Syst. Signal Pr., 27, 729$742(2012)$.

4. A. Heng, S. Zhang, A. C. Tan, and J. Mathew, Mech. Syst. Signal Pr., 23, 724-739 (2009).

5. A. K. Jardine, D. Lin, and D. Banjevic, Mech. Syst. Signal Pr., 20, 1483-1510 (2006). 